\title{
VANISHING HOMOLOGY OVER NILPOTENT GROUPS 1
}

\author{
WILLIAM G. DWYER
}

ABSTRACT. Let $\pi$ be a nilpotent group and let $M$ be a $\pi$-module. Under certain finiteness assumptions we prove that the twisted homology groups $H_{i}(\pi, M)$ vanish for all positive $i$ whenever $H_{0}(\pi, M)=0$.

The purpose of this note is to prove the following vanishing theorem:

(1) Theorem. Let $\pi$ be a finitely generated nilpotent group, and let $M$ be a $\pi$-module which is finitely generated over $\mathbf{Z}[\pi]$. Assume that $H_{0}(\pi, M)$ $=0$. Then $H_{i}(\pi, M)=0$ for all $i \geq 0$.

In the statement of this theorem, the $\pi$-module $M$ is, as usual, an abelian group equipped with a left $\pi$-action, $Z[\pi]$ is the integral group ring of $\pi$, and $H_{i}(\pi, M), i \geq 0$, are the twisted homology groups defined in [4].

With a little care, the proof below will also yield the following more general result:

(2) Theorem. Let $\pi$ be as in (1), and let $\left\{M_{s}\right\}_{s \geq 0}$ be a tower of $\pi$-modules, each of which is finitely generated over $\mathbf{Z}[\pi]$. Then if $\left\{H_{0}\left(\pi, M_{s}\right)\right\}_{s \geq 0}$ is protrivial, all the other towers $\left\{H_{i}\left(\pi, M_{s}\right)\right\}_{s \geq 0}$ for $i>0$ are protrivial too.

For a definition of the terms in this statement, and a discussion of the basic properties of towers, see [1].

Several topological applications of these theorems will be examined in forthcoming papers [2], [3]. Our statements are parallel to the results of $[5]$, although very different in detail.

The author does not know in what sense these theorems are "best possible." There are examples to show that the finite generation condition on $M$ and the nilpotency condition on $\pi$ are unavoidable, but the finite generation condition on $\pi$ may well be redundant. In fact, a simple proof of (1)

Received by the editors December 3, 1973.

AMS (MOS) subject classifications (1970). Primary 18H10; Secondary 20E 15, 20D15, 20K40.

Key words and phrases. Nilpotent group, twisted group homology.

${ }^{1}$ Research supported by NSF GP-38590. 
for arbitrary abelian $\pi$ appears at the end of this note, but the author cannot see how to generalize it.

Preliminaries. In the discussion below, a $\pi$-module $M$ such that $H_{0}(\pi, M)$ vanishes is called perfect; a module $M$ such that $H_{i}(\pi, M)$ vanishes for all $i \geq 0$ is called acyclic. Three observations will be used repeatedly:

Any quotient module of a perfect $\pi$-module is perfect.

This follows at once from the fact that $H_{0}(\pi,-)$ is right exact.

If $M^{\prime}$ is a submodule of $M, M$ is perfect, and $M / M^{\prime}$ is acyclic, then $M^{\prime}$ is perfect.

This follows at once from the long exact homology sequence of $0 \rightarrow M^{\prime}$ $\rightarrow M \rightarrow M / M^{\prime} \rightarrow 0$.

The integral group ring of a finitely generated nilpotent group is (left and right) noetherian.

This is the backbone of the argument below. A proof is indicated in [6].

Now the proof of (1) proceeds by induction on the number of central cyclic extensions needed to construct $\pi$. Consequently, we can assume that $\sigma$ is a cyclic subgroup of the center of $\pi$ and that the obvious inductive theorem is known for $\pi / \sigma$-modules. Let $M$ be a finitely generated perfect $\mathbf{Z}[\pi]$-module. We let $s$ be a generator of $\sigma$, and $T$ the endomorphism of $M$ given by $m \mapsto m-s \cdot m$ for all $m \in M$. The letter $p$ will denote the order of $\sigma$, which can be assumed either prime or infinite; if $p$ is infinity, then by convention every element of every abelian group is said to be of order $p$.

Special cases. In this paragraph we will prove that $M$ is acyclic if it has one of the following three special forms:

Type I. $T$ is injective on $M$.

Type II. $T$ is the zero map $M \rightarrow M$, and $M$ has no elements of order $p$. (This type is trivial if $p=\infty$.)

Type III. $T$ is the zero map $M \rightarrow M$, and every element of $M$ has order $p$.

If $M$ falls into one of these three classes, we will compute the $E^{2}$ term of the Lyndon spectral sequence [M]

$$
E_{p, q}^{2}=H_{p}\left(\pi / \sigma, H_{q}(\sigma, M)\right) \Rightarrow H_{p+q}(\pi, M)
$$


and show that it vanishes. Actually, the computation below will only show that

$$
H_{0}\left(\pi / \sigma, H_{j}(\sigma, M)\right)=E_{0, j}^{2}=0 \text { for all } j .
$$

The induction hypothesis, together with the easily proven fact that each $H_{j}(\sigma, M)$ is a finitely generated $\mathbf{Z}[\pi / \sigma]$-module, will then give $H_{i}\left(\pi / \sigma, H_{j}(\sigma, M)\right)=E_{i, j}^{2}=0$ for all $i, j \geq 0$.

The computation depends on explicit knowledge of the homology groups $H_{i}(\sigma, M)$, which are well known to be given as follows [4]:

Case I. $\sigma$ is finite of order $p$. Let $N$ be the endomorphism of $M$ given by $1+s+s^{2}+\cdots+s^{p-1}$.

$$
\begin{aligned}
H_{0}(\sigma, M) & =M / \operatorname{image}(T), \\
H_{2 i}(\sigma, M) & =\operatorname{kernel}(N) / \operatorname{image}(T), \quad i \geq 1, \\
H_{2 i+1}(\sigma, M) & =\operatorname{kernel}(T) / \operatorname{image}(N), \quad i>0 .
\end{aligned}
$$

Case II. $\sigma$ is infinite cyclic.

$H_{0}(\sigma, M)=M /$ image $(T), \quad H_{1}(\sigma, M)=\operatorname{kernel}(T), \quad H_{i}(\sigma, M)=0, \quad i>1$.

The computation now breaks into three parts, according to the structure of $M$. Recall that we are given $H_{0}\left(\pi / \sigma, H_{0}(\sigma, M)\right) \simeq H_{0}(\pi, M)=0$.

Type I. In this case $H_{2 i+1}(\sigma, M)=0$ for all $i \geq 0$. If $\sigma$ is infinite cyclic, $H_{2 i}(\sigma, M), i \geq 1$, is zero, so there is nothing more to show. Otherwise, if $\sigma$ is finite, each $H_{2 i}(\sigma, M), i \geq 1$, is a sub- $\pi / \sigma$-module of $H_{0}(\sigma, M)$, and so, by the argument of $(4), H_{0}\left(\pi / \sigma, H_{2 i}(\sigma, M)\right)=0$.

Type II. If $\sigma$ is infinite, there is nothing to show. If $\sigma$ is finite, then $H_{2 i}(\sigma, M)=0$ for $i \geq 1$ and $H_{2 i+1}(\sigma, M), i \geq 0$, is a quotient $\pi / \sigma$-module of $H_{0}(\sigma, M)$, and so, by (3), $H_{0}\left(\pi / \sigma, H_{2 i+1}(\sigma, M)\right)=0$.

Type III. If $\sigma$ is finite, then $H_{i}(\sigma, M) \simeq M \simeq H_{0}(\sigma, M)$ for all $i>0$, so that $H_{0}\left(\pi / \sigma, H_{i}(\sigma, M)\right)=0$. If $\sigma$ is infinite, then $H_{1}(\sigma, M) \simeq M \simeq H_{0}(\sigma, M)$, and $H_{i}(\sigma, M)=0$ for $i>1$, so the same argument works.

The general case. Let $M$ be an arbitrary perfect finitely generated $\mathbf{Z}[\pi]$-module. In order to show that $M$ is acyclic, it is enough to show that there is a finite $\pi$-filtration of $M$,

$$
0=F_{0} \subseteq F_{1} \subseteq F_{2} \subseteq \cdots \subseteq F_{k}=M
$$

such that each filtration quotient $F_{i+1} / F_{i}$ is of Type I, II, or III. Indeed, if such a filtration exists, then we show by descending induction on $i$ that 
$M / F_{i}$ is acyclic for all $0 \leq i \leq k$. The induction starts with the fact that $M / F_{k}=M / M=0$ is acyclic. Now suppose that $M / F_{i}$ is acyclic, $i>0$. There is a short exact sequence

$$
0 \rightarrow F_{i} / F_{i-1} \rightarrow M / F_{i-1} \rightarrow M / F_{i} \rightarrow 0
$$

By (3) $M / F_{i-1}$, as a quotient of $M$, is a perfect $\pi$-module. The long exact homology sequence of this short exact sequence, and the fact that $M / F_{i}$ is acyclic, show that $F_{i} / F_{i-1}$ is perfect. Since this $\pi$-module is of Type I, II, or III, it must be acyclic. Another look at the long exact homology sequence verifies that $M / F_{i-1}$ is acyclic.

Constructing such a filtration of $M$ is not hard. Let $T$ be as above, and define $F_{i}(i \geq 0)$ by

$$
F_{0}=\{0\} \subset M, \quad F_{i}=\operatorname{kernel}\left\{T^{i}: M \rightarrow M\right\}, \quad i \geq 1 .
$$

Since $\sigma$ is in the center of $\pi,\left\{F_{i} \mid i \geq 0\right\}$ is a family of $\pi$-equivariant submodules of $M$. By the noetherian condition (5), this family must have a maximal element, so, for some $K \geq 0, F_{K}=F_{K+1}$. Then $M / F_{K}$ is of Type I. If $\sigma$ is infinite cyclic, each $F_{i+1} / F_{i}$ is already of Type III, and we are done. Otherwise, if $\sigma$ is finite of order $p$, it is enough to show that each $F_{i+1} / F_{i}$ can be filtered (in a $\pi$-equivariant way) so that the filtration quotients are of Type II or III. To do this, pick $0 \leq i \leq K-1$, and define $G_{j}$ $(j \geq 0)$ by

$$
G_{0}=F_{i}, \quad G_{j}=\left\{x \in F_{i+1} \mid p^{j} x \in F_{i}\right\}_{0}
$$

Again by the noetherian condition, there is some $J \geq 0$ such that $G_{J}=$ $G_{J+1}$. Clearly $F_{i+1} / G_{J}$ is of Type II, and each $G_{j+1} / G_{j}$ is of Type III. This completes the proof.

A simple proof of the abelian case. We give a conceptual proof for arbitrary abelian $\pi$ that any perfect $\pi$-module $M$ which is finitely generated over $\mathbf{Z}[\pi]$ is acyclic. It is enough to assume that $M$ has a single generator $m$ over $\mathbf{Z}[\pi]$; induction on the number of generators, using (4), then gives the general case.

Let $I \subseteq \mathbf{Z}[\pi]$ be the augmentation ideal-the kernel of the natural epimorphism $\mathbf{Z}[\pi] \rightarrow \mathbf{Z}$. By hypothesis, $H_{0}(\pi, M)=\mathbf{Z} \otimes \mathbf{Z}[\pi]^{M=M / I} \cdot M=0$, so there must be some $r \in I$ such that $r \cdot m=m$. Since $\pi$ is abelian, left multiplication by $r$ commutes with the action of $\pi$ and so must induce the identity map $M \rightarrow M$, and therefore the identity map $H_{*}(\pi, M) \rightarrow H_{*}(\pi, M)$. 
However, again since $\pi$ is abelian, left multiplication by $r$ is easily seen to induce a natural transformation of the functor $H_{*}(\pi,-)$ into itself; this natural transformation is evidently zero on $H_{0}(\pi,-)$ and so, by the basic theorems about derived functors, must be identically zero. Consequently, the identity map $H_{*}(\pi, M) \rightarrow H_{*}(\pi, M)$ coincides with the zero map.

\section{REFERENCES}

1. A. K. Bousfield and D. M. Kan, Homotopy limits, completions, and localizations, Springer, New York, 1972.

2. W. Dwyer, Generalized convergence of the Eilenberg-Moore spectral sequence (in preparation).

3. E. Dror, Homology circles (in preparation).

4. S. Mac Lane, Homology, Die Grundlehren der math. Wissenschaften, Band 114, Academic Press, New York; Springer-Verlag, Berlin, 1963. MR 28 \# 122.

5. T. Nakayama, On modules of trivial cohomology over a finite group, Illinois J. Math. 1 (1957), 36-43. MR 18, 793.

6. D. Passman, Infinite group rings, Dekker, New York, 1971, p. 136.

DEP ARTMENT OF MATHEMATICS, YALE UNIVERSITY, NEW HAVEN, CONNECTICUT 06520 